\title{
Quantitative characterization of developing collagen gels using optical coherence tomography
}

\author{
David Levitz \\ Monica T. Hinds \\ Niloy Choudhury \\ Noi T. Tran \\ Stephen R. Hanson \\ Oregon Health \& Science University \\ Department of Biomedical Engineering \\ 3303 SW Bond Avenue \\ Mailcode $\mathrm{CH} 13 \mathrm{~B}$ \\ Portland, Oregon 97239
}

\author{
Steven L. Jacques \\ Oregon Health \& Science University \\ Department of Biomedical Engineering \\ 3303 SW Bond Avenue \\ Mailcode $\mathrm{CH} 13 \mathrm{~B}$ \\ Portland, Oregon 97239 \\ and \\ Oregon Health \& Science University \\ Department of Dermatology \\ Dermatology Research Division \\ 3181 SW Sam Jackson Park Road \\ Mailcode L468R \\ Portland, Oregon 97239
}

\begin{abstract}
Nondestructive optical imaging methods such as optical coherence tomography (OCT) have been proposed for characterizing engineered tissues such as collagen gels. In our study, OCT was used to image collagen gels with different seeding densities of smooth muscle cells (SMCs), including acellular gels, over a five-day period during which the gels contracted and became turbid with increased optical scattering. The gels were characterized quantitatively by their optical properties, specified by analysis of OCT data using a theoretical model. At $6 \mathrm{~h}$, seeded cell density and scattering coefficient $\left(\mu_{\mathrm{s}}\right)$ were correlated, with $\mu_{\mathrm{s}}$ equal to $10.8 \mathrm{~cm}^{-1} /\left(10^{6}\right.$ cells $\left./ \mathrm{mL}\right)$. Seeded cell density and the scattering anisotropy $(g)$ were uncorrelated. Over five days, the reflectivity in SMC gels gradually doubled with little change in optical attenuation, which indicated a decrease in $g$ that increased backscatter, but only a small drop in $\mu_{\mathrm{s}}$. At five days, a subpopulation of sites on the gel showed substantially higher reflectivity (approximately a tenfold increase from the first $24 \mathrm{~h}$ ). In summary, the increased turbidity of SMC gels that develops over time is due to a change in the structure of collagen, which affects $g$, and not simply due to a change in number density of collagen fibers due to contraction. (c) 2010 Society of Photo-Optical Instrumentation Engineers. [DOI: 10.1117/1.3377961]
\end{abstract}

Keywords: optical coherence tomography; scattering; optical properties; anisotropy; tissue engineering; collagen gels; hydrogels.

Paper 09395R received Sep. 3, 2009; revised manuscript received Feb. 23, 2010; accepted for publication Feb. 24, 2010; published online Apr. 28, 2010.

\section{Introduction}

The nondestructive characterization of engineered tissues has generated enormous interest recently. Frequently, tissue engineers are interested in monitoring the local microenvironment in an engineered tissue and assessing parameters, such as cell number, cell function, protein expression, and mechanical properties. Most current techniques that characterize these parameters are either destructive, require adding (often expensive) labels to the sample, or making macroscopic measurements with no possibility of assessing local variations. ${ }^{1-4}$ On the microscopic level, engineered tissue constructs often have local heterogeneities, and the patency of implanted constructs can depend on assessing these local variations.

In general, optical methods have shown great promise for nondestructively characterizing engineered tissues. One particularly promising method is optical coherence tomography (OCT), which offers several unique technological advantages for characterizing engineered tissues ${ }^{5}$. Commercial OCT systems can achieve an axial spatial resolution of $<10 \mu \mathrm{m}$ and penetrate up to $1 \mathrm{~mm}$ in highly scattering media, imaging at video-rate speeds. As a fiber-based technology, OCT can be

Address all correspondence to: Steven L. Jacques, Oregon Health \& Science University, Department of Biomedical Engineering, 3303 South West Bond Avenue, Mailcode CH13B, Portland, Oregon 97239. Tel: 503-418-9338; Fax: 503418 9311; E-mail: jacquess@ohsu.edu coupled into flexible handheld probes, allowing them to be easily incorporated into incubators.

Several recent studies have used OCT to characterize engineered tissues. Initially, Tan et al. used OCT to visualize how fibroblasts change the architecture of a 3D chitosan scaffold, ${ }^{6}$ and then to monitor cell migration, proliferation, and detachment off a calcium phosphate scaffold by trypsinization. ${ }^{7}$ Rey et al. visualized chemotaxis-induced cell migration in 3-D agarose substrates. ${ }^{8}$ OCT has also been used to visualize how cells change the porosity in poly(1-lactic acid) scaffolds ${ }^{9}$ and chitosan scaffolds. ${ }^{10}$

These papers clearly demonstrate the ability of OCT to characterize features such as cells (or a cluster of cells) on a weakly scattering background of an imaged tissue construct. However, as the tissue construct develops, gel compaction, cell proliferation, and matrix remodeling change the size and density of scatterers in the sample. At this stage, the construct becomes a highly scattering medium, in which the cells can no longer be resolved. Properly characterizing mature tissue constructs just prior to implantation will be important for ensuring successful outcomes, but given the highly scattering nature of mature constructs, making such assessments is quite challenging.

Moreover, the bulk of the work in characterizing engineered tissue constructs using OCT has been qualitative im-

1083-3668/2010/15(2)/026019/11/\$25.00 @ 2010 SPIE 
$24 \mathrm{hr}$

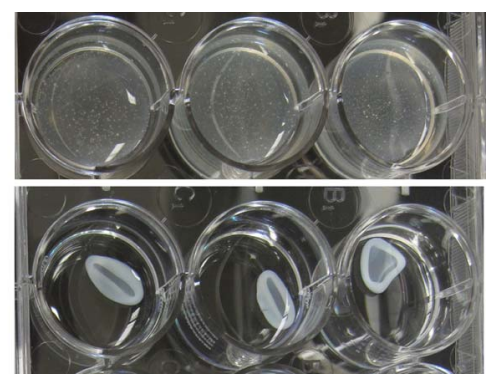

Fig. 1 Photographs of triplicate SMC collagen gels at $24 \mathrm{~h}$ and five days. Well diameter $=2.26 \mathrm{~cm}$.

age analysis, based primarily on identifying features of interest (i.e., a cell) by a subjective operator. Given the qualitative nature of these methods, systematically surveying large volumes of data $\left(\sim 1 \mathrm{~cm}^{3}\right)$ imaged at micron resolution is both cumbersome and impractical. However, OCT data contain quantitative information on the optical scattering properties of the imaged sample. The optical properties of interest here are the scattering coefficient $\mu_{\mathrm{s}}\left[\mathrm{cm}^{-1}\right]$ and anisotropy factor $g$ [dimensionless], ${ }^{11}$ which provide a measure of the density and size of scattering particles in the medium, respectively. With proper theoretical modeling, it is possible to describe the depth-dependent OCT signal as a function of $\mu_{\mathrm{s}}$ and $g$, as well as the system parameters. ${ }^{12}$ By fitting a depth-dependent OCT signal that is representative of local nanoliter volumes in the sample to a theoretical model, it is possible to determine the local scattering properties of the sample. This approach was used to evaluate the optical properties of one of the most common types of engineered tissues, collagen gels, and characterize their development when seeded with cells.

Collagen gels are one of the simplest types of engineered tissues, in which cells are suspended in a 3-D collagen I matrix. Over time, the cells pull on the collagen fibrils causing gel compaction, wherein the gel becomes smaller, with decreased water content, and visibly more opaque. Some mesenchymal cells, such as fibroblasts and smooth muscle cells (SMCs), also contract and remodel the collagen matrix. ${ }^{13} \mathrm{An}$ example of SMC-seeded collagen gels at $24 \mathrm{~h}$ and five days is shown in Fig. 1. Note that the 24-h gels are large and nearly transparent, while the five-day gels have contracted and have a cloudy appearance.

In this paper, collagen gels are imaged with different cellseeding densities over a five-day period and the data are used to measure their optical properties. Over time, the reflectivity of the gels increased while the attenuation did not change, which degraded the ability to visualize structures such as cells. This reflectivity increase was due to a decrease in anisotropy $g$, which caused increased backscatter of light. No such changes were observed in acellular gels. In each set of gels imaged at five days, reflectivity values followed a bimodal distribution, with the major population of gel sites showing a doubling of reflectivity and a sizeable subpopulation showing a roughly tenfold increase in reflectivity relative to the first $24 \mathrm{~h}$. Time dynamics of the gels showed that these changes in optical properties were gradual. A careful analysis of the optical properties measured during the earliest time point showed that a correlation existed between cell density and $\mu_{\mathrm{s}}$, but that cell density and $g$ were uncorrelated. Finally, mechanisms that could be responsible for the observed changes in optical properties are discussed.

\section{Theory}

The theory of light propagation in low-coherence imaging systems used to process the data in this paper was derived from the inverse Monte Carlo method. ${ }^{12}$ For a homogeneous turbid medium characterized by a scattering coefficient $\mu_{\mathrm{s}}$, an anisotropy factor $g$, an absorption coefficient $\mu_{\mathrm{a}}$, and a refractive index $n$, the depth-dependent OCT reflectance signal $R(z)$ can be described as an exponential decay

$$
R(z)=\rho e^{-\mu z},
$$

where the reflectivity $\rho$ is the fraction of light delivered to the focus that is backscattered by the focal volume of tissue within the solid angle of collection of the microscope's objective lens, and the attenuation $\mu$ describes the signal attenuation as light propagates to and from the focal volume. Specifically,

$$
\rho=\mu_{s} b(g) \Delta z
$$

and

$$
\mu=2 G\left[\mu_{s} a(g)+\mu_{a}\right],
$$

in which $\Delta z$ is the axial resolution of the imaging system and $G$ is a geometry factor accounting for the extra pathlength caused by off-axis propagation during delivery and collection by the objective lens. Analysis has shown that for an objective lens with numerical aperture (NA), the parameter $G$ can be approximated by $1 / \cos \left[\sin ^{-1}(\mathrm{NA})\right]$. For low-NA imaging systems frequently used in OCT, $G$ is close to 1.0 (the exact value of $G$ for high NA systems has been discussed ${ }^{12}$ ). The product $\mu_{\mathrm{s}} \Delta z$ is the number of scattering events that occur within the focal volume, and the parameter $b(g)$ represents the fraction of light scattered within the focal volume that is backscattered into solid angle of collection of the objective lens. For a medium with scattering phase function $p(\theta), b(g)$ is given by

$$
b(g)=\int_{\pi-\sin ^{-1}(\mathrm{NA})}^{\pi} p(\theta) 2 \pi \sin (\theta) d \theta .
$$

In our analysis, a Henyey-Greenstein phase function ${ }^{14}$ is assumed. One of the terms in Eq. (3) is $a(g), 0<a<1$, that decreases the effectiveness of scattering when the scattering is forward directed such that photons, despite multiple scattering, still manage to reach the focus and cannot be distinguished from the unscattered light. For highly anisotropic media, $a(g)$ has the effect of reducing the observed attenuation in the OCT signal. The function $a(g)$ can be approximated by a numerical expression determined from Monte Carlo simulations 


$$
a(g)=1-\exp \left(\frac{(1-g)^{0.6651}}{0.1555}\right) .
$$

Experiments and numerical simulations have shown that this model is valid for confocal reflectance microscopes, which include both reflectance confocal microscopy (RCM) and OCT operated in focus-tracking mode. ${ }^{12}$ The coherence gating in OCT improves on RCM by rejecting photons whose path length in the tissue does not match the round-trip path length to/from the focus, which yields about a 1000-fold noise reduction that enables deeper imaging. ${ }^{12}$ Preliminary data show that this model is valid approximately up to a (one-way) optical depth of $\mu_{\mathrm{s}} z=3$, before multiple scattering begins to considerably interfere with the signal.

For this model to be valid, it is necessary to convert the interferometric OCT envelope (in arbitrary units) into dimensionless units of reflectance $R(z)$, i.e., the fraction of delivered light that is collected and detected by the microscope. To do this, the signal is calibrated against the peak signal from a known interface, such as an oil-glass interface, or against the reflectivity of an optical scattering phantom with known optical properties. This calibration is crucial in order to map the observations $\mu$ and $\rho$ into the optical properties $\mu_{\mathrm{s}}$ and $g$.

\section{Materials and Methods}

\subsection{Cell Culture}

All cell culture materials were purchased from GIBCO (Invitrogen, Carlsbad, California) unless otherwise noted. The experiments used primary SMCs isolated from a single baboon carotid artery that was chemically digested. Passage 9 SMCs were used in preparing collagen gels. The cells were fed with SMC growth medium (SGM), consisting of minimum essential medium (MEM) supplemented with $10 \%$ fetal bovine serum, $1 \%$ L-glutamine, and $1 \%$ penicillin-streptomycin.

\subsection{Collagen Gel Preparation}

All collagen gels were prepared using the same basic protocol. Soluble calf skin collagen (part no. 150026, MP Biomedicals, Solon, Ohio) was dissolved in $0.02 \mathrm{~N}$ acetic acid at a concentration of $4.85 \mathrm{mg} / \mathrm{mL}$. In preparing the collagen gels, eight parts collagen $(\mathrm{pH}=2.5)$ were mixed with two parts $5 \times$ MEM and one part 0.2 M NaOH (Sigma, Sigma-Aldrich, St. Louis, Missouri), with $\mathrm{NaOH}$ titrated until the $\mathrm{pH}$ was neutral as indicated by a color change in phenol red in the solution. Immediately thereafter, five parts SGM containing the predetermined number of cells were added to the mixture and aliquoted as $1-\mathrm{mL}$ samples into a 12 -well plate (Corning, Lowell, Massachusetts). The weakly scattering suspension thermally gelled for $1 \mathrm{~h}$ at $37^{\circ} \mathrm{C}$. Thereafter, $1 \mathrm{~mL} \mathrm{SGM}$ was added to feed each gel. Overall, 24 gels were prepared at each cell concentration $\left(3.8 \times 10^{5}, 1 \times 10^{6}\right.$, and 2.1 $\times 10^{6}$ cells $/ \mathrm{mL}$ ), which is denoted below as a "set" of collagen gels. Additionally, a set of acellular gels was also prepared and imaged. Acellular collagen gels were prepared in an identical manner to the SMC collagen gels, except that cells were absent from the mixed solutions.

Gels that were cultured for longer than $24 \mathrm{~h}$ were detached from the well at $24 \mathrm{~h}$ with a spatula and allowed to further compact. These gels were fed $1 \mathrm{~mL}$ SGM every two days. At each imaging time point, three gels were removed from their wells and imaged by OCT. After OCT imaging, each sample was fixed for subsequent histological examination.

In addition to the four sets of collagen gels described above, optical property data acquired with a different OCT system are also presented. The two other sets of gels (one seeded with SMCs and one acellular) were imaged with a different OCT system at the 24-h and five-day time points. There were minor differences between these two sets and the four above, namely, the NA was 0.08 and the initial collagen density was $2 \mathrm{mg} / \mathrm{mL}$. The OCT images, gel compaction data, and histology images for these two sets were nearly identical to the data presented here and are thus not shown. Altogether, imaging of the six sets of collagen gels was done over a span of 15 months.

\subsection{OCT Imaging}

A time-domain OCT imaging system was setup as previously described. ${ }^{15}$ Briefly, a 9-mW broadband light source operating at $1310 \mathrm{~nm}$ (B\&W Tek, Newark, Delaware) with a 94-nm full width at half maximum (FWHM) bandwidth was coupled into a fiber-based Michelson interferometer. A rapid scanning optical delay line ${ }^{16}$ varied the delay in the reference arm. A $10 \times$ microscope objective (Newport, Irvine, California) focused the light on the sample. Both scanning mirrors in the reference and sample arms ( $X$ scanning) were from GSI Lumonics (Bedford, Massachusetts). The interference signal between the backreflected light from the sample and reference arms was measured using an InGaAs photodiode (New Focus, Santa Clara, California). The OCT system was controlled through a data acquisition card (National Instruments, Austin, Texas) using software written in Matlab (MathWorks, Natick, Massachusetts). As the system scanned axially, the interference of the sample and reference arms yielded an oscillating signal. The Hilbert transform was performed in software postacquisition using Matlab to specify the envelope of this oscillating signal, which was proportional to the reflected electric field. Squaring this envelope yielded the signal proportional to reflected intensity.

During OCT imaging, the sample was mounted on a glass slide and immersed in MEM to maintain hydration throughout imaging. Each gel was imaged at five sites, with the sample repeatedly imaged 10-12 times at each imaging site. The sample orientation was randomized between imaging sites. In the 10-12 images acquired at each site, the translation stage on which the sample was mounted was manually moved toward the objective lens by $50 \mu \mathrm{m}$ to a position $\left(z^{\prime}\right)$. An axial OCT scan versus depth $z$ was obtained at each lens position, yielding a signal $R\left(z, z^{\prime}\right)$. In separate experiments using an oil-glass interface that could be positioned at different depths, a focus function was specified, $F\left(z, z^{\prime}\right)$, which accounted for the variation in response as the position of a structure of interest $(z)$ deviated from the central focus position $\left(z^{\prime}\right)$. $F\left(z, z^{\prime}\right)$ showed that the portions of each image in the range $z=z^{\prime} \pm 25 \mu \mathrm{m}$ experienced only minor defocusing to roughly $81 \%$ of the maximum value. Moreover, $50 \mu \mathrm{m}$ was chosen as the focus shift distance since $25 \mu \mathrm{m}$ is $\sim 1 / 8$ of the depth of focus in our OCT system $(196 \mu \mathrm{m})$. The portions of each image in the range $z=z^{\prime} \pm 25 \mu \mathrm{m}$ provided the strongest signals and were stitched together to yield a final image. Defo- 
cusing effects were negligible in comparison to the speckle fluctuations in the signal, which spanned an order of magnitude. In this manner, a quasi-focus tracking image was prepared, appropriate for the theoretical OCT model.

\subsection{OCT Image Processing Algorithm}

The OCT data from all samples were processed in a similar manner. A single, Matlab-based algorithm for evaluation of optical properties from the data was organized in the following separate modular steps:

1. The OCT data at each imaging site were read into Matlab and stitched together to form a quasi-focus tracked image. Using user-defined landmarks as points of reference (typically the air-sample interface or the corners of the sample), images taken at a single site were stitched together.

2. The data from a stitched image were then calibrated against the signal from a known reference. Initially, the data were calibrated against a mineral oil-glass interface $\left(\rho=1.826 \times 10^{-4}\right)$. However, polystyrene sphere-agarose gel phantom data acquired during the same period of collagen gel imaging revealed that sphere phantoms are a more stable calibration method, and thus all the relevant collagen gel images were cross-calibrated against sphere data from 210 regions of interest (ROIs) from 30 imaging sites in six phantoms.

3. To align OCT images, the most superficial pixel of the gel was found at each lateral $x$ position, and the image was aligned by shifting each A-scan axially. For samples with the top surface located at the air-MEM interface, this edge was found by fitting a parabola to the interface pixels. For samples submerged in MEM with an irregular surface, the user manually drew a line along the gel surface using the computer mouse. Once the sample surface was identified, the A-scans were shifted accordingly.

4. The user subdivided each calibrated, stitched, aligned image into five ROIs along the $x$ direction for fitting. The $x z$ dimensions of each ROI were approximately $90 \times 450 \mu \mathrm{m}$. The average signal over $x$ at each $z$ position yielded $R_{\text {avg }}(z)$.

5. For each ROI, $\ln \left(R_{\text {avg }}\right)$ versus $z$ was fit to Eq. (1) using a linear fit. A weighted-least-squares fitting routine was used, with the weight at each $z$ pixel being inversely proportional to the variance of the signal through all the $x$ pixels at that depth. In addition to the fitted parameters $\mu$ and $\rho$, and their respective confidence intervals, goodness-of-fit statistics - the mean pixel error and the adjusted regression coefficient $R^{2}$-were documented for each ROI.

\subsection{Gel Compaction}

Collagen gel compaction was monitored in the gels at eight time points and expressed as a percent of the initial gel volume $(1 \mathrm{~mL})$. The volume of the gels was indirectly measured via the volume of the media in the well that could be aspirated after compaction, i.e., gel volume $=2 \mathrm{~mL}$ minus the aspirated volume, with the $2 \mathrm{~mL}$ coming from a $1-\mathrm{mL}$ gel fed with $1 \mathrm{~mL}$ SGM. The gel compaction was measured on SMC gels with three cell seeding densities and an initial collagen density of $2.43 \mathrm{mg} / \mathrm{mL}$. Calibration experiments showed that this method overestimated the collagen gel volume by $<50 \mu \mathrm{L}$.

\subsection{Histology}

After imaging, samples were fixed overnight in $10 \%$ neutral buffered formalin and stored in $70 \%$ ethanol at $4{ }^{\circ} \mathrm{C}$. The samples were embedded in paraffin, and $5-\mu \mathrm{m}$ sections were cut and stained with hematoxylin and eosin (H\&E) on glass slides. Sections of the selected samples were cut from positions starting at $1 / 2$ the radius of the sample toward the center, in $50-\mu \mathrm{m}$ increments. The histological sections were cut so they would expose a cross section of the gels, but they did not exactly match the imaging planes in the OCT data since the gel orientation was randomized between the imaging sites. The histological sections were imaged by phase-contrast microscopy (Optiphot, Nikon, Tokyo, Japan) at $20 \times$.

\subsection{Data Analysis and Statistics}

Each linear fit of $R_{\text {avg }}(z)$ data at each ROI resulted in a $(\mu, \rho)$ pair. The $(\mu, \rho)$ data were plotted as a scatter plot against a grid of iso- $\mu_{\mathrm{s}}$ and iso- $g$ contours generated by the model of Eqs. (1)-(3). The contours map experiment to theory. Alternatively, the data were assessed by looking at the distributions of fitted $(\mu, \rho)$ values. For both $\mu$ and $\rho$, the probability density functions (PDFs), $f(\mu)$ and $f(\rho)$, were computed by binning $\log _{10}(\mu)$ and $\log _{10}(\rho)$ values from an individual group into histograms made of Gaussian shaped kernels. Treating $\mu$ and $\rho$ as statistically independent random variables, a joint PDF was computed using $f(\mu, \rho)=f(\mu) f(\rho)$. Finally, the peaks of the $(\mu, \rho)$ joint PDFs were mapped back to $\left(\mu_{\mathrm{s}}, g\right)$ with the a priori knowledge of the basic system parameters $\Delta z$ and NA using Eq. (2) and (3).

To determine the accuracy and precision of the algorithm used in this paper, preliminary experiments were conducted to statistically characterize the fitting results. Specifically, intraROI, inter-ROI, inter-site, and inter-sample coefficients of variation were calculated for sphere phantoms, 24-h collagen gels, and five-day collagen gels (Table 1). Data in Table 1 are expressed as means \pm standard deviation over $n$ samples, and are in units of percent. The intra-ROI variability describes the reproducibility of 10 measurements of $\mu$ and $\rho$ on the same ROI. The inter-ROI variability describes the variation of the mean $\mu$ and $\rho$ of each ROI between five ROIs on the same site on a gel. The inter-site variability describes the variation of the mean data of five ROIs on a site between five sites on the same phantom/collagen gel. The inter-sample variability describes the variation of the mean data of five sites on one phantom between three different phantoms (or collagen gels). Because of this averaging, the inter-site and inter-sample variability in Table 1 are lower than the inter-ROI measurements. The sphere data in Table 1 illustrate the accuracy and reproducibility of the measurements, the 24-h collagen gel data illustrate the inherent variations in the gel preparation, and the five-day collagen gel data illustrate the inherent variations in gel development. The resolution of the algorithm for determining optical properties was specified as the FWHM of the PDF for either $\mu$ or $\rho$ values. The FWHM resolution in $\mu$ was finite at $20 \mathrm{~cm}^{-1}$. For $\rho$, the resolution scaled with the fitted results, with the FWHM being $\sim 20 \%$ of the mean. In terms of accuracy, the algorithm output was shown to overestimate the theoretically predicted values for both $\mu$ and $\rho$ by $\sim 10 \%$. 
Levitz et al.: Quantitative characterization of developing collagen gels...

Table 1 Statistics describing the accuracy and reproducibility of the algorithm. Listed are the coefficients of variation for intra-ROI, inter-ROI (intra-site), inter-site (intra-sample), and inter-sample measurement in both $\mu$ and $\rho$, which are expressed in percent as means \pm standard deviations over $n$ samples. Data are given for sphere phantoms, as well as the key time points of 1 and $5 \mathrm{~d}$ during collagen gel development.

\begin{tabular}{|c|c|c|c|c|c|c|c|c|c|}
\hline \multirow{2}{*}{$\begin{array}{l}\text { Sample } \\
\text { type }\end{array}$} & \multicolumn{3}{|c|}{ Sphere phantoms } & \multicolumn{3}{|c|}{ Collagen gels, $1 \mathrm{~d}$} & \multicolumn{3}{|c|}{ Collagen gels, $5 \mathrm{~d}$} \\
\hline & $n$ & $\mu$ & $\rho$ & $n$ & $\mu$ & $\rho$ & $n$ & $\mu$ & $\rho$ \\
\hline Intra-ROI ${ }^{\mathrm{a}}$ & 84 & $5.1 \pm 4.2$ & $9.1 \pm 5.1$ & 63 & $35.4 \pm 24.3$ & $19.1 \pm 6.1$ & 63 & $19.0 \pm 12.8$ & $19.9 \pm 11.3$ \\
\hline Inter-ROI & 30 & $5.3 \pm 2.7$ & $13.3 \pm 5.7$ & 45 & $39.9 \pm 24.8$ & $30.6 \pm 13.9$ & 46 & $38.8 \pm 25.4$ & $23.4 \pm 13.8$ \\
\hline Inter-site & 6 & $4.0 \pm 2.6$ & $12.0 \pm 3.9$ & 9 & $28.6 \pm 9.5$ & $30.7 \pm 11.6$ & 9 & $35.2 \pm 9.6$ & $30.3 \pm 21.8$ \\
\hline Inter-sample & 3 & $4.8 \pm 1.9$ & $17.5 \pm 5.9$ & 3 & $25.4 \pm 14.1$ & $34.7 \pm 14.5$ & 3 & $47.9 \pm 28.4$ & $53.1 \pm 41.6$ \\
\hline
\end{tabular}

${ }^{a}$ Outliers that are $>3$ standard deviations from the mean (corresponded to $<4 \%$ of data) were excluded.

\section{Results}

\subsection{Ability to Visualize Cells in OCT Images of Collagen Gels Decreases with Time}

Representative images of collagen gels with different cell seeding densities (acellular, $3.8 \times 10^{5}, 1 \times 10^{6}$, and 2.1 $\times 10^{6}$ cells $\left./ \mathrm{ml}\right)$ at $6 \mathrm{~h}, 24 \mathrm{~h}$, and five days are shown in Figs. 2(a)-2(1), with corresponding histology for the SMC gels in Figs. 2(m)-2(u). The bright line on the top of some of the OCT images represents the air-MEM interface. Overall, the OCT images appeared rather spatially uniform at any given time point, with the only visualized structures being bright spots that presumably represent individual cells or clusters of cells. Such bright spots on a weakly scattering background can be seen in the 6-h images of SMC gels [Figs. 2(b)-2(d)]. The density of these small "bright" spots increases with increasing cell seeding density. The bright spots were $\sim 20 \mu \mathrm{m}$ in size, comparable to SMCs. At $24 \mathrm{~h}$ [Figs. 2(e)-2(h)] a few more of these bright spots can be seen in the $2.1 \times 10^{6}$ gels [Fig. 2(h)], and to a lesser extent in the $1 \times 10^{6}$ and 3.8 $\times 10^{5}$ gels [Figs. 2(f) and $\left.2(\mathrm{~g})-2(\mathrm{G})\right]$. At 5 d [Figs. 2(i) $\left.-2(\mathrm{l})\right]$, the images look different. In the $3.8 \times 10^{5}$ gels [Fig. 2(j)], there are a few bright spots that are difficult to resolve. The $1 \times 10^{6}$ and $2.1 \times 10^{6}$ gels [Figs. $2(\mathrm{k})$ and $2(\mathrm{l})$ ] show a superficial high signal region that decays. For both gel conditions, it is not possible to resolve any cells in the foreground, as the bright spots have blended into the signal rich background. Note that because of the high superficial signal and the attenuation, the images appeared featureless and uniform. These gels have entered a scattering regime in which qualitative image visualization does not yield much useful information and in which quantitative image processing techniques are needed. In all the acellular samples [Figs. 2(a), 2(e), and 2(i)], there were larger, modestly brighter, and darker regions in the background of all the images caused by heterogeneity in scattering from the collagen, which did not appear to change much over time. Histologically, the H\&E sections in Figs. 2(m)-2(u) show an increase in cell density over time for each of the three different seeding densities.

\subsection{Collagen Gels Exhibit an Increase in Reflectivity/ Decrease in Anisotropy over Time}

The optical properties of the developing collagen gels from a single representative set are shown on a scatter plot in Fig. 3.
Also plotted on the scatter plot is a grid that maps theoretical $\left(\mu_{\mathrm{s}}, g\right)$ values to the observed $(\mu, \rho)$ pairs. Here, the initial collagen density was $2.43 \mathrm{mg} / \mathrm{mL}$ and the initial cell density was $1 \times 10^{6}$ cells $/ \mathrm{mL}$. Figure 3 compares SMC and acellular gels at two key time points of one and five days, with each datum point representing a $(\mu, \rho)$ pair from a single ROI. In assessing the SMC and acellular gels at $24 \mathrm{~h}$, the relative proximity of these two groups suggests that the scattering is dominated by the collagen fibrils, not the cells, which make sense given the small volume fraction the cells occupy. Also, note that the reflectivity in the SMC gels more than doubled from one to five days, whereas the corresponding acellular gels had only a modest increase in reflectivity. There was a large variance in attenuation values in all the samples. This feature has been observed consistently over multiple sets of collagen gels and results in poorer ability to resolve $\mu$ in collagen gels, in contrast to sphere phantoms (Table 1). Moreover, neither the SMC nor acellular gels experienced a major shift in attenuation over five days.

A summary of the optical property results from multiple sets of collagen gels is detailed in Table 2, which lists the peak values and range $( \pm 1 / e$ or \pm 0.37 of peak value) for each $\mu$ and $\rho$ of the joint PDFs. These ranges provide an estimate of how $\mu$ and $\rho$ values were distributed around the joint PDF peaks. In comparing multiple sets of gels, the optical properties follow a general trend of starting out at low reflectivity and low attenuation at times $<24 \mathrm{~h}$, and gradually increasing reflectivity over five days, approximately twofold, while attenuation remained relatively constant. These observed $(\mu, \rho)$ pairs corresponded to $\mu_{\mathrm{s}}$ values that varied greatly, from 7 to $93 \mathrm{~cm}^{-1}$, while $g$ values were consistently closely centered around 0.90 in the first $24 \mathrm{~h}$. At five days, $\mu_{\mathrm{s}}$ values of SMC gels consistently decreased $30-50 \%$, while $g$ decreased to $\sim 0.75$. Moreover, at five days, there was also a consistent subpopulation of data with higher reflectivity values, which were approximately tenfold the reflectivity observed in the first $24 \mathrm{~h}$. Thus, at five days the reflectivity of collagen gels followed a bimodal distribution as can be seen in the reported peak ranges in Table 2. Note that the peak $\rho$ value is consistently closer to the lower boundary than the upper boundary, which suggests that although most of the data were centered around the peak, there was always a sizeable subpopulation with even higher $\rho$ values. In contrast to the SMC gels, acellular gels from set 4 and set 6 had very minor 


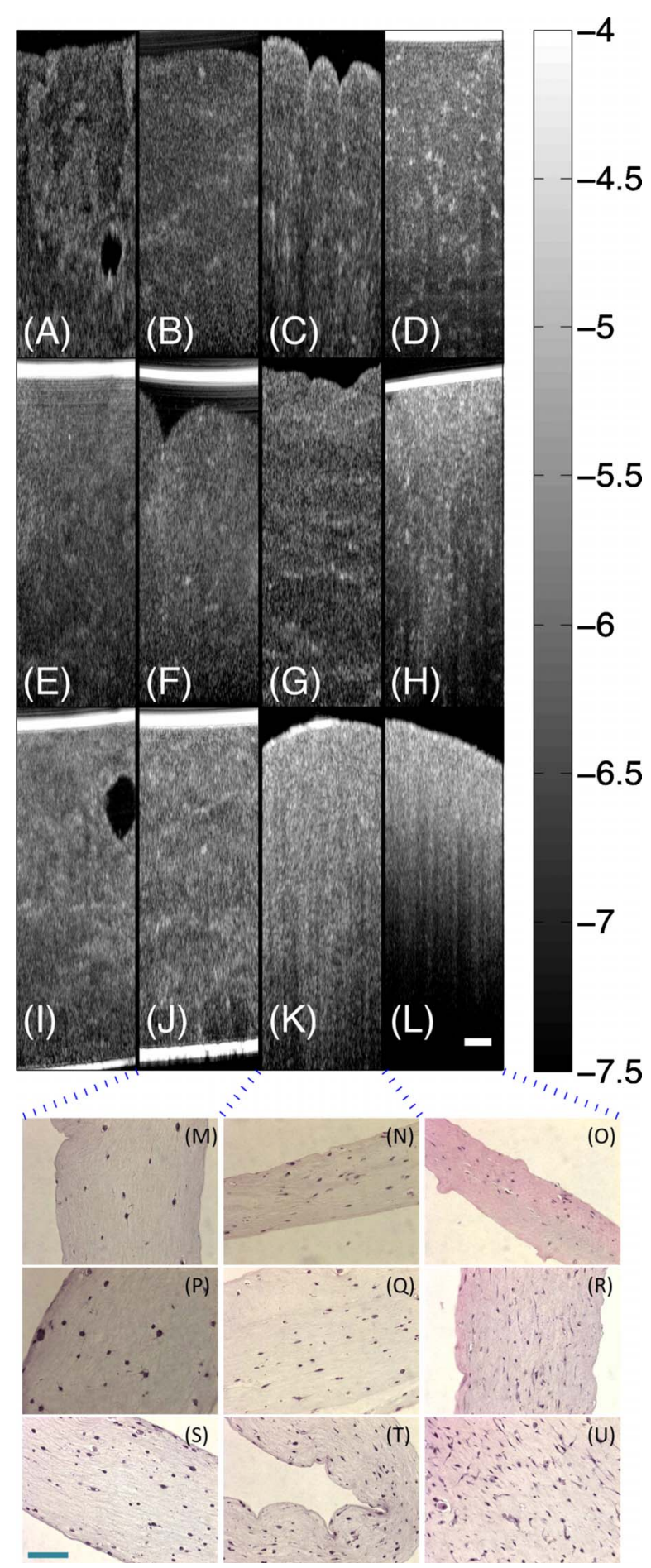

Fig. 2 (a-l) Representative OCT images at (a-d) 6 h, (e-h) 24 h, and (i-l) five days for acellular gels and three different cell seeding densities. Acellular gels are shown in $(\mathrm{a}, \mathrm{e}, \mathrm{i})$; gels seeded with 3.8 $\times 10^{5}$ cells $/ \mathrm{mL}$ are shown in $(\mathrm{b}, \mathrm{f}, \mathrm{j}) ; 1 \times 10^{6}$ cell $/ \mathrm{mL}$ gels are shown in $(\mathrm{c}, \mathrm{g}, \mathrm{k})$; and $2.1 \times 10^{6} \mathrm{cell} / \mathrm{mL}$ gels are shown in $(\mathrm{d}, \mathrm{h}, \mathrm{l})$. Note that other than a few bright spots, the images (particularly at five days) are rather featureless, making visual characterization difficult. The bright spots are presumed to be cells or clusters of cells. Gray scale represents $\log _{10}(\rho)$. (m-u) Corresponding histology for the three seeding densities of SMC gels: $(\mathrm{m}-\mathrm{O}) 6 \mathrm{~h},(\mathrm{p}-\mathrm{r}) 24 \mathrm{~h}$, and $(\mathrm{s}-\mathrm{u}) 5 \mathrm{~d}$. Gels seeded with $3.8 \times 10^{5}$ cells $/ \mathrm{mL}$ are shown in $(\mathrm{m}, \mathrm{p}, \mathrm{s}) ; 1$ $\times 10^{6}$ cell $/ \mathrm{mL}$ gels are shown in $(\mathrm{n}, \mathrm{q}, \mathrm{t})$; and $2.1 \times 10^{6} \mathrm{cell} / \mathrm{mL}$ gels are shown in $(\mathrm{o}, \mathrm{r}, \mathrm{u})$. The histology images show an increase in cell density took place for the three sets of gels over time. Bar $=100 \mu \mathrm{m}$.

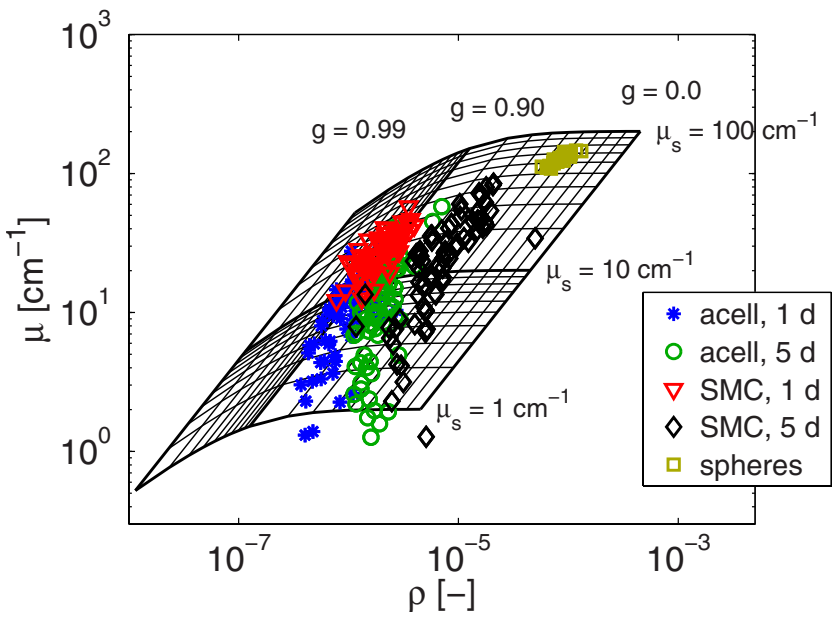

Fig. 3 Representative scatter plot showing the overall trends in $(\mu, \rho)$ data from both SMC and acellular gels at one and five days. Each point represents the fit results from an individual ROI. Note that at $1 \mathrm{~d}$ there was overlap between the SMC and acellular gel data, but at five days the $(\mu, \rho)$ data from SMC and acellular gels have drifted apart. The data displayed here correspond to sets 2 and 4 in Table 1, with an initial collagen density was $2.43 \mathrm{mg} / \mathrm{mL}$ and an initial cell density (passage 9 ) of $1 \times 10^{6} \mathrm{cell} / \mathrm{mL}$.

shifts toward either higher or lower reflectivity, respectively. Nonetheless, despite a large variance in $\mu$, the increase in $\rho$ of collagen gels over time was both consistent and reproducible. Thus, although the spatial heterogeneity of collagen structures in the acellular gels remained constant over time, the subpopulation of higher $\rho$ values across the various sets of fiveday SMC gels suggests that some spatial heterogeneity developed in the SMC gels over time.

\subsection{Time Dynamics of Collagen Gels}

Although Fig. 3 shows the overall shift in the local optical properties of the collagen gels, it is also worthwhile to visualize the time dynamics of the optical properties. However, because the shift in the $(\mu, \rho)$ data over time is slow and many data points lie on top of one another, the actual 2-D distribution of $\mu$ and $\rho$ values is presented as the joint PDF. A representative set of the calculated joint PDFs at $6 \mathrm{~h}, 24 \mathrm{~h}$, three days, and five days are plotted superimposed on a grid in Figs. 4(a)-4(d), showing the gradual increase in reflectivity over five days. Figures 4(a) and 4(b) shows that there are not many changes in the optical properties over the first $24 \mathrm{~h}$. However, from one to five days [Figs. 4(b)-4(d)] the center of the PDF shifted to the right toward higher reflectivity, without changing much in attenuation. There was also a substantial subpopulation in the five-day time point that had an even higher reflectivity (lower anisotropy), similar to the other data sets (Table 2).

Additionally, the peaks of the joint PDFs shown in Figs. 4(a)-4(d) were mapped from $(\mu, \rho)$ space back to $\left(\mu_{\mathrm{s}}, g\right)$ space. Plots of $\mu_{\mathrm{s}}$ and $g$ versus time for eight time points spanning five days are displayed in Figs. 4(e) and 4(f). Error bars represent the $1 / e$ width of the joint PDF at the iso- $\mu_{\mathrm{s}}$ and iso- $g$ lines of the PDF peak. The $g$ versus time curve [Fig. 4(f)] shows that over one to five days, there was a moderate but steady decrease in anisotropy. A similar moderate but 
Levitz et al.: Quantitative characterization of developing collagen gels...

Table 2 Summary of optical properties from multiple sets of collagen gels, from the earliest time point $(6 \mathrm{~h}$ in sets $1-3,24 \mathrm{~h}$ in sets 4 and 5$)$ to the final time point ( $5 \mathrm{~d}$ for all sets). For both $\mu$ and $\rho$, we report both the peak of the joint PDF and the range over which the joint PDF $>1 / e^{*}$ maximum. Square brackets denote concentration. Collagen density units are $\mathrm{mg} / \mathrm{ml}$; cell density units are cells/ml; $\mu$ and $\mu_{\mathrm{s}}$ units are $\mathrm{cm}^{-1} ; \rho$ and $g$ units are dimensionless.

\begin{tabular}{|c|c|c|c|c|c|c|c|c|}
\hline Set & No. gels & {$[\text { collagen }]_{\mathrm{t}=0}$} & {$[\text { cell }]_{\mathrm{t}=0}$} & Cell passage no. & $\mu_{\text {early }}$ & $\mu_{\text {final }}$ & $\mu_{\text {early }}$ pk. range & $\mu_{\text {final }}$ pk. range \\
\hline 1 & 24 & 2.43 & $3.8 \times 10^{5}$ & 9 & 16.22 & 15.31 & $4.37-35.48$ & $6.38-36.31$ \\
\hline 2 & 24 & 2.43 & $1 \times 10^{6}$ & 9 & 30.90 & 30.55 & $16.79-49.55$ & $6.68-80.35$ \\
\hline 3 & 24 & 2.43 & $2.1 \times 10^{6}$ & 9 & 43.15 & 53.09 & 17.99-76.86 & $24.55-84.14$ \\
\hline 4 & $3^{b}$ & 2.43 & 0 & NA & 10.47 & 15.14 & $4.57-29.51$ & $4.37-32.36$ \\
\hline $5^{a}$ & 6 & 2 & $1 \times 10^{6}$ & 5 & 59.57 & 48.98 & $14.62-117.49$ & $17.99-108.39$ \\
\hline $6^{a}$ & 6 & 2 or $5^{c}$ & 0 & NA & 38.46 & 60.26 & $9.12-92.26$ & $26.00-89.13$ \\
\hline Set & $\rho_{\text {early }}$ & $\rho_{\text {final }}$ & $\rho_{\text {early }}$ pk. range & $\rho_{\text {final }}$ pk. range & $\mu_{\text {s-early }}$ & $\mu_{\text {s-final }}$ & gearly & $g_{\text {final }}$ \\
\hline 1 & $1.38 \times 10^{-6}$ & $2.95 \times 10^{-6}$ & $5.19-29.2 \times 10^{-7}$ & $2.16-3.94 \times 10^{-6}$ & 10.50 & 8.19 & 0.896 & 0.756 \\
\hline 2 & $2.16 \times 10^{-6}$ & $5.37 \times 10^{-6}$ & $1.01-3.51 \times 10^{-6}$ & $2.60-21.1 \times 10^{-6}$ & 21.69 & 16.55 & 0.919 & 0.775 \\
\hline 3 & $3.27 \times 10^{-6}$ & $4.79 \times 10^{-6}$ & $7.16-73.3 \times 10^{-7}$ & $2.40-29.5 \times 10^{-6}$ & 29.23 & 33.66 & 0.910 & 0.889 \\
\hline 4 & $7.76 \times 10^{-7}$ & $1.80 \times 10^{-6}$ & $4.37-11.78 \times 10^{-7}$ & $1.11-3.09 \times 10^{-6}$ & 7.16 & 8.85 & 0.913 & 0.847 \\
\hline $5^{a}$ & $1.15 \times 10^{-6}$ & $2.48 \times 10^{-6}$ & $7.16-21.4 \times 10^{-7}$ & $1.30-5.01 \times 10^{-6}$ & 37.30 & 25.75 & 0.878 & 0.690 \\
\hline $6^{a}$ & $4.95 \times 10^{-7}$ & $3.05 \times 10^{-7}$ & $1.93-12.6 \times 10^{-7}$ & $1.35-5.62 \times 10^{-7}$ & 28.32 & 93.47 & 0.927 & 0.985 \\
\hline
\end{tabular}

aNote that sets 5 and 6 were imaged with a different OCT system.

bote 3 samples were imaged at eight different time points.

${ }^{\mathrm{C}}$ The initial collagen concentration in the gels imaged at $1 \mathrm{~d}$ and $5 \mathrm{~d}$ were 2 and $5 \mathrm{mg} / \mathrm{mL}$, respectively.

steady decrease is also seen in the $\mu_{\mathrm{s}}$ versus time plot [Fig. $4(\mathrm{e})]$. Note the nonlinear mapping of $\left(\mu_{\mathrm{s}}, g\right)$ to $(\mu, \rho)$ that occurs at $g$ values $>0.9$, such that reflectivity can increase and attenuation can show no change while $g$ and $\mu_{\mathrm{s}}$ both decrease.

Macroscopically, the SMC collagen gels showed a moderate but consistent rate of gel compaction, i.e., the rate of decrease in collagen gel volume as the cells pull on the fibrils was steady [Fig. 4(g)]. Gel compaction was assessed at the eight imaging time points by quantifying the volume of media in the gel well just before imaging. The volume of gels from each of the three SMC groups shrunk to about 20-30\% of their original volume. These data agreed with end point measurements by Cummings et al. ${ }^{17}$

\subsection{Cell Density Correlates with Scattering Coefficient of Collagen Gels}

When analyzing the optical property results, the 6-h data were closest to the zero time point and thus had minimal change in both the cells and the collagen. In plotting the peaks of the joint PDFs on a grid [Fig. 5(a)], these $(\mu, \rho)$ pairs were colocated on an iso- $g$ line, and that their $\mu_{\mathrm{s}}$ value increased with cell concentration. The corresponding $\mu_{\mathrm{s}}$ and $g$ values for the four points were backtracked, as in Figs. 4(e) and 4(f), and plotted against the cell seeding density [Figs. 5(b) and 5(c)]. Included in Figs. 5(b) and 5(c) are linear fits of both $\mu_{\mathrm{s}}$ and $g$ against cell seeding density. The fit results showed that $\mu_{\mathrm{s}}$ was highly correlated with cell concentration $\left(R^{2}=0.998\right)$ and in- creased linearly with cell density at a rate of $10.8 \mathrm{~cm}^{-1} /\left(10^{6}\right.$ cells $\left./ \mathrm{mL}\right)$ plus a $7.0-\mathrm{cm}^{-1}$ offset that represents scattering from collagen alone [Fig. 5(b)]. Moreover, the $7.0 \mathrm{~cm}^{-1}$ measured from $2.43 \mathrm{mg} / \mathrm{mL}$ acellular collagen corresponds to a $\mu_{\mathrm{s}}$ increase of $2.88 \mathrm{~cm}^{-1} /(\mathrm{mg} / \mathrm{mL})$ of collagen density. A similarly high correlation $\left(R^{2}=0.985\right)$ between cell density and $\mu_{\mathrm{s}}$ was obtained by correlating cell-like structures (i.e., "bright spots") counted manually in the 6-h gel images (data not shown). In contrast to $\mu_{\mathrm{s}}, g$ remained flat relative to the concentration [Fig. 5(c)] and was uncorrelated $\left(R^{2}\right.$ $=0.035)$. Such an increase in $\mu_{\mathrm{s}}$ without a change in $g$ is expected for a static system with an increasing scattering particle (i.e., cell) density. However, the imaged collagen gels are dynamic over five days, and no such clear cut correlations existed between either $\mu_{\mathrm{s}}$ or $g$ and cell concentration at any of the later time points. This suggests that, over five days, the contraction process involved a fundamental change in the overall collagen gel architecture.

\section{Discussion}

In this paper, OCT was used to image developing collagen gels at different time points and to measure the optical properties from the images by fitting the OCT signal to a theoretical model. The results showed that over time, there was a consistent twofold increase in reflectivity with little change in the attenuation in four separate sets of experiments with various cell seeding densities (Table 2, Figs. 3 and 4). Also, consistently present in each set of gels imaged at five days was a 

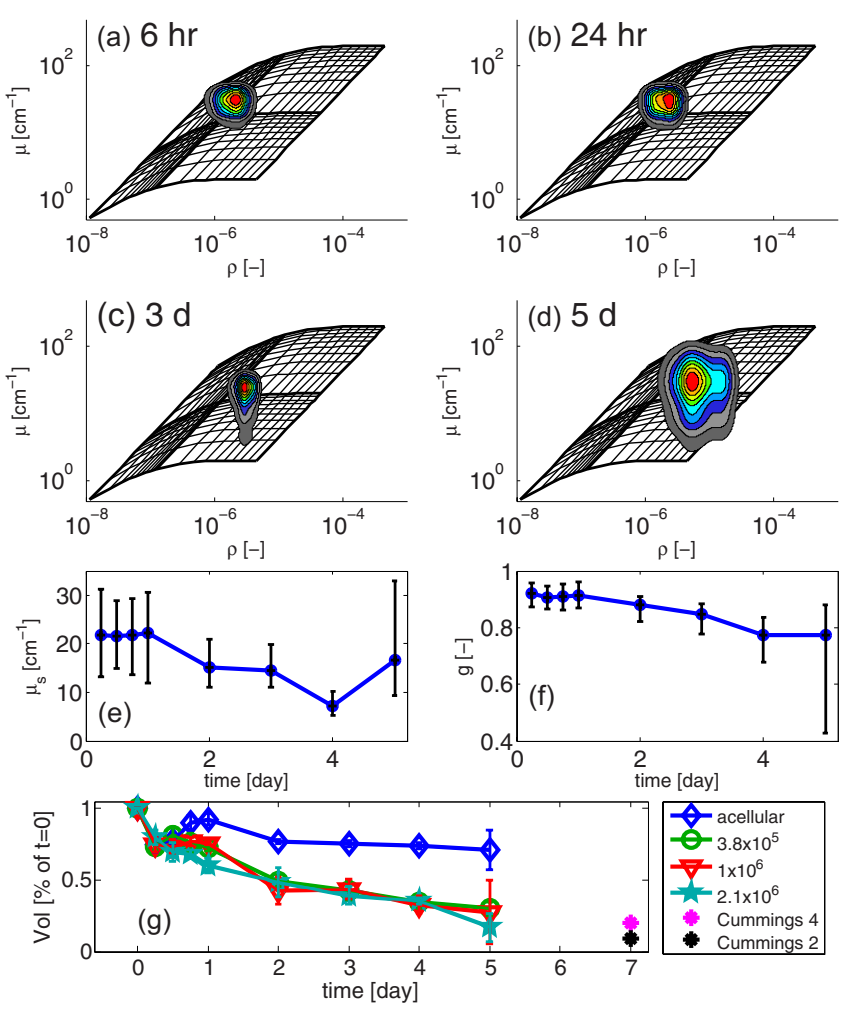

Fig. 4 Log-log plots showing 2-D PDFs of collagen gels at (a-d) $6 \mathrm{~h}$, $24 \mathrm{~h}, 3$ days, and 5 days. Also on each plot is a grid that shows how theoretical optical properties $\left(\mu_{\mathrm{s}}, g\right)$ map to $(\mu, \rho)$ in our OCT system. Over time, the center of the PDF moves to the right, showing an increase in reflectivity. Moreover, the 5-d PDF also has a subpopulation at higher $\rho$ values, indicating that a subpopulation of the ROls had a higher reflectivity. (e, f) Plots of peaks in $(\mu, \rho)$ space that were mapped back to $\left(\mu_{\mathrm{s}}, g\right)$, plotted versus time. The displayed data in (a-f) correspond to set 2 in Table 1, in which the initial collagen density was $2.43 \mathrm{mg} / \mathrm{mL}$, and the initial cell density (passage 9) was $1 \times 10^{6}$ cells $/ \mathrm{mL}$. (g) Gel compaction curve for the gels versus time. These data correspond to sets 1-4 in Table 1; the first four legend entries represent initial cell concentrations in units of cells $/ \mathrm{ml}$. Cummings' gels had a $1 \times 10^{6}$ cell $/ \mathrm{mL}$ seeding density, and an initial collagen density of either 4 or $2 \mathrm{mg} / \mathrm{mL}^{17}$

sizeable subpopulation of sites on the gel with a higher reflectivity, roughly tenfold greater than the first $24 \mathrm{~h}$. These trends in attenuation $\mu$ and reflectivity $\rho$ correspond to a decrease in anisotropy $g$ and a moderate decrease in scattering coefficient $\mu_{\mathrm{s}}$, suggesting an overall decrease in the size of the scattering particles.

Several models have been proposed for measuring optical properties from OCT signals. The first model is the singlescattering (SS) model, in which the signal is assumed to follow an exponential decay $R(z) \propto \exp \left[-2\left(\mu_{\mathrm{s}}+\mu_{\mathrm{a}}\right) z\right]$ $\approx \exp \left(-2 \mu_{\mathrm{s}} z\right)$, since $\mu_{\mathrm{s}} \gg \mu_{\mathrm{a}}$ for most tissues at near-infrared wavelengths. Despite similarities between the SS and $\mu-\rho$ models used here, our results illustrate several flaws in using the SS model for measuring optical properties. First, the $\mu-\rho$ model fits two parameters that map back to two unknowns, $\mu_{\mathrm{s}}$ and $g$, whereas the SS model only fits one parameter $\left(\mu_{\mathrm{s}}\right)$. However, our results repeatedly showed that the shift with time in optical properties of the collagen gels was more clearly observed in $g$, not $\mu_{\mathrm{s}}$. Second, the main difference in
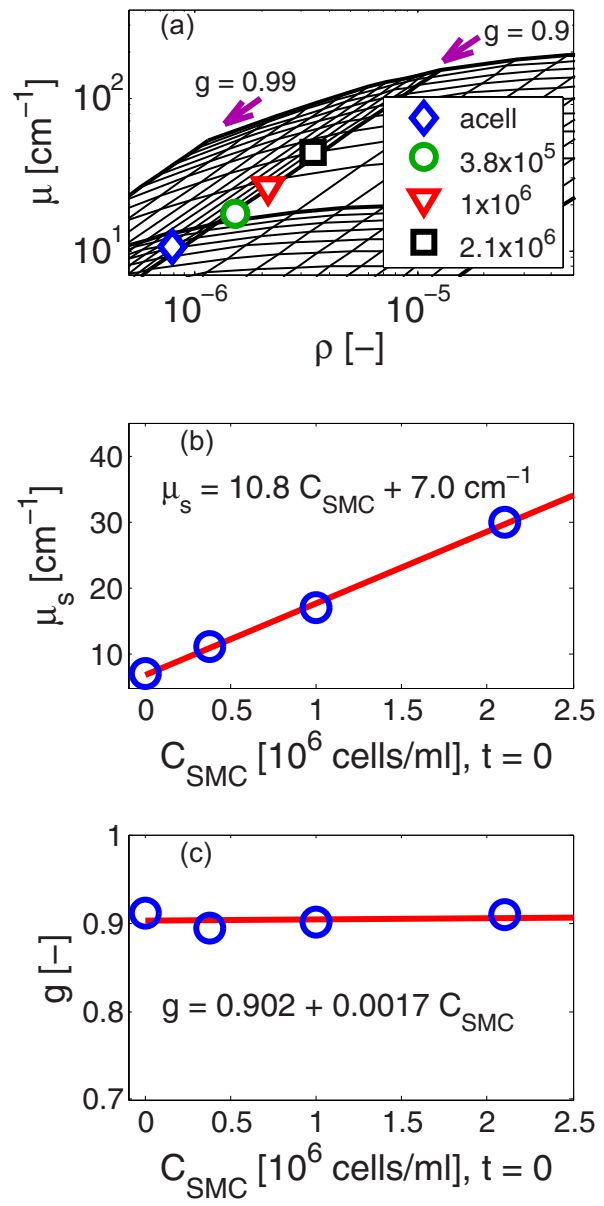

Fig. 5 (a) Peak of $\mu$ and $\rho$ PDFs from $6 \mathrm{~h}$ gels plotted on grid. Note how all the points line up on the same iso-g line, with increasing $\mu_{\mathrm{s}}$ values. These data correspond to sets $1-4$ in Table 1 ; the units on the legend are in cells per milliliter. Plots of (b) $\mu_{\mathrm{s}}$ and (c) $g$ data calculated from the $(\mu, \rho)$ PDFs for the four cell densities, along with corresponding linear fits. Note that $\mu_{\mathrm{s}}$ increases linearly with cell density, while $g$ does not change.

the attenuation term of the two models is the factor $a(g)$, which describes how scattered light can still reach the focus despite multiple scattering. It is $a(g)$ that makes the mapping from $\mu_{\mathrm{s}}$ to $\mu$ nonlinear (and hence nontrivial) at high $g$ values, to which the $6-24$ h collagen gel $(\mu, \rho)$ data mapped.

Most of the work on fitting optical properties from OCT imaging has focused on differentiating different types of atherosclerotic plaques. ${ }^{18-20}$ Each of these studies used a different theoretical model. Van der Meer et al. ${ }^{19}$ used the SS model and showed differences in $\mu_{\mathrm{s}}$ between normal arteries and atherosclerotic plaques. Levitz et al. ${ }^{18}$ used the extended Huygens-Fresnel (EHF) model ${ }^{21}$ and showed differences in anisotropy between normal arteries and atherosclerotic plaques. The EHF model has the added advantage of accounting for multiple scattering and defocusing; that is, it is capable of evaluating both $\mu_{\mathrm{s}}$ and a measure of anisotropy similar to $g$ without the need for the (time consuming) quasi-focus tracking scheme implemented in our study. However, the EHF model is highly nonlinear and thus requires a computationally burdensome algorithm that does not always converge to a solution. Alternatively, there are other models that have been 
used to measure optical properties from highly scattering samples. The model used by $\mathrm{Xu}$ et al. ${ }^{20}$ is very similar to the $\mu-\rho$ model used here, but with key differences. First, the $a(g)$ is missing from this model, and second, $\rho$ is represented as the backscattering coefficient $\mu_{\mathrm{b}}$, with no nonlinear mapping back to $g$.

Similar to our work, $\mathrm{Xu}$ et al. ${ }^{22}$ characterized cytochrome oxidase activity in astrocytes suspended in agarose gels by measuring changes in $\mu_{\mathrm{a}}$ via the fitted attenuation, but using the SS model with its inherent limitations. Nonetheless, it is also worth noting that in their cell-agarose samples, a negligible amount of scattering and absorption from the agarose contributed to the signal; thus, all the scattering and absorption measured could be attributed to the cells. Figure 5 shows that cells are mild scatterers and that a very high density is needed to obtain a measureable change in attenuation. The attenuation values measured by $\mathrm{Xu}$ et al. ${ }^{22}$ required very high cell seeding densities $\left(\sim 10^{7}-10^{8}\right.$ cells $\left./ \mathrm{mL}\right)$, which is in general agreement with our results.

The experiments presented here highlight several advantages to measuring local optical properties of samples using OCT. The method is nondestructive, which makes continuous and time-lapse monitoring of the tissue gels feasible. Because this OCT method depends on endogenous contrast, no labels are needed, which has the added benefits of both preserving the sample unperturbed in its native environment and reducing the costs associated with fluorescent labels. Moreover, the visual information contained in the OCT images complements the quantitative optical property data. And although these experiments were destructive in nature-samples were prepared, removed from the sterile incubator and imaged, and then fixed for histological analysis - one of OCT's primary advantages is that it is a fiber-based technology that can be sterilized and integrated into the incubator for continuous, time-lapse monitoring. ${ }^{6}$ The adaptation of confocal mosaicing technology ${ }^{23}$ to OCT will enable rapid surveying of large sample volumes. These features, if successfully implemented, could enable automated nondestructive evaluation of large volumes of engineered tissues.

In this study, samples were imaged and images were further divided into volume regions of about $90 \times 10$ $\times 450 \mu \mathrm{m}(x y z)$, or $0.4 \mathrm{~nL}$. This resulted in a rather large number of ROIs with volumes on the nanoliter scale, which partly affected the analyses. Implicit in the definition of $\mu_{\mathrm{s}}$ and $g$ is the assumption of averaging over some volume. The nanoliter volumes assessed here approach the size limit over which these properties are defined, but nonetheless, it is still possible to define optical properties within these volumes. These small volumes, from which optical properties are measured, are a direct consequence of the spatial resolution and field of view of OCT images. Indeed, ROI volumes on the nanoliter scale have been used by others when measuring optical properties from OCT data. ${ }^{18-20,22}$

One consequence of these small sampling volumes is an increase in the variance of the optical properties, which may simply reflect the intrinsic heterogeneity of the samples on this size scale. The variance in optical properties of acellular gels was much larger than those in SMC gels (Figs. 3 and 4, Table 2), paradoxically suggesting that acellular gels were less homogeneous than SMC gels. However, this is not the com- plete picture. A careful examination of the acellular gels with the naked eye showed that some regions are cloudier than others, confirming millimeter-scale heterogeneity, seen in the OCT images and observed in the optical property data. Moreover, the histological sections [Figs. 2(m)-2(u)] showed that within the field of view of an OCT or histology image, the cells were generally uniformly distributed. Noting that cells are mild scatterers but with a higher reflectivity than the background collagen, the scattering in the SMC gels from the "uniformly" distributed cells contrasted with the collagen background and shifted the optical properties accordingly. However, in the acellular gels, all the scattering was due to the slowly varying background, which explains the increased variance observed in the acellular gels. Indeed, perhaps the large spatial variability in collagen distribution may offer clues as to why some regions in tissue-engineered implants are at increased risk of mechanical failure. ${ }^{24}$

The optical properties at five days showed a consistent increase in $\rho$ with little change in $\mu$, which correspond to a decrease in anisotropy $g$ with a modest decrease in $\mu_{\mathrm{s}}$. Such changes in optical properties suggest a decrease in the overall size of the scattering particles, not an increase in particle density, which would increase $\mu_{\mathrm{s}}$, and hence increase both $\mu$ and $\rho$. In attempting to identify which microstructural changes caused this shift in optical properties, we (initially) proposed four potential reasons: increased collagen density, increased cell density, SMC contraction (i.e., a change in cell morphology), and collagen remodeling. Further analyses of some of the data presented here rules out increases in collagen and cell density, and preliminary results (not shown) suggest that collagen remodeling is responsible for the changes in optical properties.

To test if increased collagen density (i.e., gel compaction) caused the twofold increase in reflectivity, the collagen density was plotted against both $\mu$ and $\rho$ for the different collagen gel samples and the correlation coefficient was calculated. There was little to no correlation between collagen density and optical properties: for $\rho$ versus collagen density, $R^{2}<0.2$; for $\mu$ versus collagen density, $R^{2}<0.06$ (data not shown). These poor correlations suggest that collagen density was not the primary cause of the measured shifts in optical properties. Furthermore, the changes in optical properties caused by changes in collagen density were very subtle $\left[\mu_{\mathrm{s}}\right.$ $\left.=2.88 \mathrm{~cm}^{-1} /(\mathrm{mg} / \mathrm{mL})_{\text {collagen }}\right]$, and are well below the $14-\mathrm{cm}^{-1}$ algorithm resolution for $\mu_{\mathrm{s}}$ at $g=0.9$. Such an increase in $\mu_{\mathrm{s}}$ with little change in $g$ does not explain the shift in optical properties over time observed in our data, namely, a decrease in $g$ a modest decrease in $\mu_{\mathrm{s}}$. Also, data from set 6 (Table 2), in which higher density $(5 \mathrm{mg} / \mathrm{mL})$ acellular gels were imaged at five days, suggests that gel compaction did not cause the change in optical properties, because the characteristic increase in reflectivity (with no change in attenuation) that was consistently observed in the SMC gels was absent. Of note, we do not expect that extracellular matrix laid down by SMCs over the five-day incubation period contributed significantly to the change in optical properties, because previous work has shown the matrix synthesized by SMCs embedded in both collagen and fibrin gels to make up $<1 \%$ of the total collagen content. ${ }^{25,26}$ 
Additionally, the correlation between the optical properties and cell density was checked. An increase in cell density may be caused either by cell proliferation and/or compounded by gel compaction. However, an alamar blue assay on three extra sets of gels did not show an increase in cell metabolism over five days, suggesting no proliferation took place (data not shown). The result is in agreement with previously published results. ${ }^{27}$ Nonetheless, to confirm whether cell density correlates with optical properties, cells were manually counted in 15 histological H\&E sections for each gel (three analogous sites in five sections separated by $50 \mu \mathrm{m}$ ), which were normalized by the area of the collagen in that section. These cell density counts were compared to both the $\mu$ and $\rho$ values for that gel, and the correlation coefficient $R^{2}$ was calculated. The correlations between the cell density and both $\mu$ and $\rho$ were rather weak. For $\mu$ versus cell density, $R^{2}=0.25$, whereas for $\rho$ versus cell density $R^{2}=0.34$ (data not shown). These weak correlations, together with the 6-h data showing how cell density correlates with $\mu_{\mathrm{s}}$ but not $g$ (Fig. 5) suggest that although an increase in cell density contributed partly to an increase in $\rho$, it was not the primary reason for the twofold increase in $\rho$ shown in the data.

The data showed that over time, there was an overall decrease in anisotropy factor $g$ of the collagen gels, as well as a moderate decrease in scattering coefficient $\mu_{\mathrm{s}}$. The decrease in $\mu_{\mathrm{s}}$ seems a bit counterintuitive because it is expected for there to be an overall increase in the density of scattering particles. However, treating the scattering particles in the gels as discretely sized microspheres, Mie theory provides some additional insight. ${ }^{11}$ Mie theory states that the relationship between the particle size and scattering cross section is highly nonlinear. Thus, consider two scattering solutions, one made with small microspheres and the other with large microspheres, but where in both cases the spheres occupy the same volume fraction. It is obvious that the small sphere solution will have a smaller $g$ value than the large sphere solution. However, these two scattering media will also have different $\mu_{\mathrm{s}}$ values, with the small sphere solution having a smaller $\mu_{\mathrm{s}}$ than the large sphere solution. Thus, if the increase in the number of small scattering particles (as indicated by the higher reflectivity) was caused by a breakdown of larger particles, one would expect a moderate decrease in the $\mu_{\mathrm{s}}$ along with a decrease in $g$. Such a scenario supports the hypothesis that remodeling of the collagen matrix into small fibril fragments caused the observed changes in optical properties.

\section{Conclusion}

In this study, OCT was used to image development in several sets of collagen gels with different cell seeding densities, as well as acellular gels, over a five-day period. After the first $24 \mathrm{~h}$, changes in the collagen gels made structures (i.e., cells) impossible to resolve. To characterize the samples quantitatively, the optical properties of the gels were determined using an image-processing algorithm that fit the OCT signal from a region of interest to a theoretical model. In doing so, the observed fit results (attenuation $\mu$ and reflectivity $\rho$ ) were mapped back to the optical properties, the scattering coefficient $\mu_{\mathrm{s}}$ and the scattering anisotropy factor $g$. Within the first $24 \mathrm{~h}$, the optical properties of the SMC and acellular gels were similar (Figs. 3 and 4), suggesting that the optical prop- erties were dominated by scattering from collagen fibrils. Over time, collagen gels with SMCs consistently exhibited a twofold increase in reflectivity, while acellular gels did not (Figs. 3 and 4, Table 2). The shift in optical properties toward higher reflectivity was gradual. A sizeable subpopulation of the SMC gels at five days had reflectivity values roughly tenfold higher than those in the first $24 \mathrm{~h}$ were consistently observed in five sets of gels. Such a shift corresponded to a decrease in $g$ and an overall decrease in the size of the scattering particles. Additionally, at the earliest imaging time point (6 h, Fig. 5), cell seeding density was strongly correlated to $\mu_{\mathrm{s}}\left(R^{2}>0.99\right)$, but not to $g\left(R^{2}=0.02\right)$. All in all, the data suggest that SMCs change the architecture of the collagen gel and that OCT can visualize and quantify these changes.

\section{References}

1. T. Guda, M. Appleford, S. Oh, and J. L. Ong, "A cellular perspective to bioceramic scaffolds for bone tissue engineering: the state of the art," Curr. Top. Med. Chem. 8(4), 290-299 (2008).

2. S. T. Ho and D. W. Hutmacher, "A comparison of micro CT with other techniques used in the characterization of scaffolds," Biomaterials 27(8), 1362-1376 (2006).

3. C. W. Jones, D. Smolinski, A. Keogh, T. B. Kirk, and M. H. Zheng, "Confocal laser scanning microscopy in orthopaedic research," Prog. Histochem. Cytochem. 40(1), 1-71 (2005).

4. D. B. Smith, M. S. Sacks, P. M. Pattany, and R. Schroeder, "Highresolution magnetic resonance imaging to characterize the geometry of fatigued porcine bioprosthetic heart valves," J. Heart Valve Dis. 6(4), 424-432, (1997).

5. C. Mason, J. F. Markusen, M. A. Town, P. Dunnill, and R. K. Wang, "The potential of optical coherence tomography in the engineering of living tissue," Phys. Med. Biol. 49(7), 1097-1115 (2004).

6. W. Tan, A. Sendemir-Urkmez, L. J. Fahrner, R. Jamison, D. Leckband, and S. A. Boppart, "Structural and functional optical imaging of three-dimensional engineered tissue development," Tissue Eng. 10(11/12), 1747-1757 (2004).

7. W. Tan, A. L. Oldenburg, J. J. Norman, T. A. Desai, and S. A. Boppart, "Optical coherence tomography of cell dynamics in threedimensional tissue models," Opt. Express 14(16), 7159-7171 (2006).

8. S. M. Rey, B. Povazay, B. Hofer, A. Unterhuber, B. Hermann, A. Harwood, and W. Drexler, "Three- and four-dimensional visualization of cell migration using optical coherence tomography," J. Biophotonics 2(6-7), 370-379 (2009).

9. Y. Yang, A. Dubois, X. Qin, J. Li, A. El Haj, and R. K. Wang, "Investigation of optical coherence tomography as an imaging modality in tissue engineering," Phys. Med. Biol. 51(7), 1649-1659 (2006).

10. P. O. Bagnaninchi, Y. Yang, N. Zghoul, N. Maffulli, R. K. Wang, and A. J. El Haj, "Chitosan microchannel scaffolds for tendon tissue engineering characterized by optical coherence tomography," Tissue Eng. 13(2), 323-331 (2007).

11. C. F. Bohren and D. R. Huffman Absorption and Scattering of Light by Small Particles, Wiley, Hoboken, NJ (1983).

12. S. L. Jacques, D. Levitz, R. Samatham, D. S. Gareau, N. Choudhury, and F. Truffer, "Light scattering in confocal reflectance microscopy," in Biomedical Applications of Light Scattering, A. Wax, Ed., pp. 171-191, McGraw-Hill, New York (2009).

13. W. D. McMillan, B. K. Patterson, R. R. Keen, and W. H. Pearce, "In situ localization and quantification of seventy-two-kilodalton type IV collagenase in aneurismal, occlusive, and normal aorta," J. Vasc. Surg. 22(3), 295-305 (1995).

14. L. G. Henyey and J. L. Greenstein, "Diffuse radiation in the galaxy," Astrophys. J. 93(1), 70-83 (1941).

15. N. Choudhury, G. Song, F. Chen, S. Matthews, T. Tschinkel, J. Zhen, S. L. Jacques, and A. L. Nuttall, "Low coherence interferometry of the cochlear partition," Hear. Res. 220(1), 1-9 (2006).

16. A. Rollins, S. Yazdanfar, M. Mulkarni, R. Ung-Arunyawee, and J. Izatt, "In vivo rate optical coherence tomography," Opt. Express 3(6), 219-229 (1998). 
17. C. L. Cummings, D. Gawlitta, R. M. Nerem, and J. P. Stegemann, "Properties of engineered vascular constructs made from collagen, fibrin, and collagen-fibrin mixtures," Biomaterials 25(17), 36993706 (2004).

18. D. Levitz, L. Thrane, M. H. Frosz, P. Andersen, C. B. Andersen, J. Valanciunaite, J. Swartling, S. Andersson-Engels, and P. R. Hansen "Determination of optical scattering properties of highly-scattering media in optical coherence tomography images," Opt. Express 12(2), 249-259 (2004).

19. F. J. van der Meer, D. J. Faber, D. M. B. Sassoon, M. C. Aalders, G. Pasterkamp, and T. G. van Leeuwen, "Localized measurement of optical attenuation coefficients of atherosclerotic plaque constituents by quantitative optical coherence tomography," IEEE Trans. Med. Imaging 24(10), 1369-1376 (2005).

20. C. Xu, J. M. Schmitt, S. G. Carlier, and R. Virmani, "Characterization of atherosclerosis plaques by measuring both backscattering and attenuation coefficients in optical coherence tomography," J. Biomed. Opt. 13(3), 034004 (2008).

21. L. Thrane, H. T. Yura, and P. E. Andersen, "Analysis of optical coherence tomography systems based on the extended Huygens-Fresnel principle," J. Opt. Soc. Am. A 17(3), 484-490 (2000).
22. X. Xu, R. K. Wang, and A. El Haj, "Investigation of changes in optical attenuation of bone and neuronal cells in organ culture or 3 dimensional constructs in vitro with optical coherence tomography: relevance to cytochrome-oxidase monitoring," Eur. Biophys. J. 32(4), 355-362 (2003).

23. D. S. Gareau, Y. Li, B. Huang, Z. Eastman, K. S. Nehal, and M. Rajadhyaksha, "Confocal mosaicing microscopy in Mohs skin excisions: feasibility of rapid surgical pathology," J. Biomed. Opt. 13(5), 054001 (2008).

24. S. L. Mitchell and L. E. Niklason, "Requirement for growing tissueengineered vascular grafts," Cardiovasc. Pathol. 12(2), 59-64 (2003.)

25. B. C. Isenberg, C. Williams, and R. T. Tranquillo, "Small-diameter artificial arteries engineered in vitro," Circ. Res. 98(1), 25-35 (2006).

26. J. J. Ross and R. T. Tranquillo, "ECM gene expression correlates with in vitro tissue growth and development in fibrin gel remodeled by neonatal smooth muscle cells," Mat. Biol. 22(6), 477-490 (2003).

27. J. P. Stegemann and R. M. Nerem, "Altered response of vascular smooth muscle cells to exogenous biochemical stimulation in twoand three-dimensional culture," Exp. Cell Res. 283(2), 146-155 (2003). 